\title{
miR-27a-3p attenuates induce autophagy-related cell death by suppressing Inhibitor of growth family 5 in cholangiocarcinoma
}

\section{Ming WAN}

Second Affiliated Hospital of Harbin Medical University

\section{Fu-min Zhang}

Daqing Oilfield General Hospital

\section{Peng-cheng Kang}

Second Affiliated Hospital of Harbin Medical University

\section{Xing-ming Jiang}

Second Affiliated Hospital of Harbin Medical University

yunfu cui ( $\nabla$ yunfu_cui@163.com )

Second Affiliated Hospital of Harbin Medical University

\section{Research}

Keywords: microRNAs, autophagy, cholangiocarcinoma, Inhibitor of growth family

Posted Date: May 13th, 2020

DOI: https://doi.org/10.21203/rs.3.rs-27745/v1

License: (c) (i) This work is licensed under a Creative Commons Attribution 4.0 International License. Read Full License 


\section{Abstract}

Background MicroRNAs (miRNAs) are abnormally expressed in human tumors, including cholangiocarcinoma (CCA). miR-27a-3p was observed up-regulated in CCA, but its functions in CCA are largely unknown.

Methods CCK8 assay, Colony formation assays and Ki-67 staining was employed to detect the cell growth. The autophagy and proliferation relative-protein analyzed by western blot. The immunofluorescence staining was applied to analyze the expression level of LC3 I/II. Tumor xenografts was used to test the role of miR-27a-3p. Luciferase reporter assay, western bolt and qRT-PCR showed the relationship between miR-27a-3p and ING5.

Results miR-27a-3p expression was increased in human CCA tissues. Inhibition of miR-27a-3p suppressed the proliferative capacity of CCA cells, silencing of miR-27a-3p dramatically induced cell death and suppressed tumor growth in vivo. The proteins, such as Beclin-1, p62, p21, p-p53, CDK4 and CDK6, were decreased upon miR-27a-3p inhibitor transfection. Western blot assay and immunofluorescence analysis were showed the induced-autophagy after transfecting with miR-27a-3p or inhibitor of growth family 5 (ING5) in RBE. ING5 as a direct miR-27a-3p target in CCA. Co-transfect of miR$27 a-3 p$ and ING5 can reverse CCA cell death which induced by miR-27a-3p inhibitor alone.

Conclusions miR-27a-3p promotes oncogenesis of CCA by triggering autophagy-related cell death by interacting with ING5 directly.

\section{Introduction}

Cholangiocarcinoma (CCA) is a serious cancer disease, affecting human health[1].This disease is a lethal form of cancer that develops as a result of the neoplastic transformation of biliary epithelial cells (BEC). Cholangiocarcinoma is associated with poor prognosis and its incidence rate has continued to increase globally [2]. Because of its high malignancy and low 5-year survival rate (10\%), it has become an important factor that seriously affects the quality of life[3]. At present, the main treatment methods for CCA are surgery, chemotherapy and radiotherapy, but the 5-year survival rate is still very low[4]. For the reason above, a new biomarker and therapeutic method for CCA is largely needed.

MicroRNAs (miRNAs) are a widely regulated human disease that exists in a wide range of diseases, such as cardiovascular disease, liver cancer, hypertension, brain diseases, and so on. However, the study of miRNA in cholangiocarcinoma is still largely unknown. A previous study implicated miR-27a-3p in the development of cardiovascular, kidney, and other diseases. Also, a study suggested that miR-27a could promote ineffective lysosomal clearance in the hippocampi of rats after chronic brain hypoperfusion. However, there are few relevant reports on cholangiocarcinoma.

Previous studies have showed that autophagy is a genera role in many diseases, such as ischemic myocardial injury[5], osteoarthritic [6] and CCA [7]. Lv P et al. showed miR-373 inhibits autophagy and 
promotes apoptosis of CCA cells by ULK1[8]. Ma et al suggest that miR-124 induces autophagy-related cell death in CCA cells by direct targeting EZH2 [9]. However, the role of autophagy in the progression of miR-27a-3p in CCA is not fully understood, and the down- or up- stream factors that control autophagy require to better understood.

Inhibitor of growth family 5 (ING5) is a member of the ING protein family, ING5 regulates many diseases such as cardiovascular disease, especially cancers. ING5 contains a motif known as PHD-finger; the motif is commonly found in proteins that are involved in chromatin remodeling [10].The ING5 protein binds to p53 and is part of the p53-dependent regulatory pathway[11]. Also, downregulation or loss of ING5 expression is observed frequently in various types of cancer. Cui et al showed that ING5 is a tumor suppresser gene that functions in the progression of breast cancer mediated by miR-24 [12]. Zhao QY et al demonstrated that ING5 inhibits EMT in breast cancer by suppressing PI3K/Akt pathway [13] .Despite this, the role of ING5 and miR-27a-3p in CC remain unclear.

Herein, we show that miR-27a-3p had an oncogenic function in cholangiocarcinoma. ING5 has been proved to have an anti-cancer effect in multiple carcinomas. We demonstrate that miR-27a-3p direct targets ING5 expression in CCA. Through suppressing ING5, miR-27a-3p enhances autophagy signaling pathway to facilitate the progression of CCA. Our newly revealed mechanism could offer a novel potential treatment target for CCA.

\section{Materials And Methods Patient information}

The Clinical Research Ethics Committee of Harbin Medical University approved the current study and animal experiments were performed as per the Guide for the Care and Use of Laboratory Animals. A total of 30 samples and $31 \mathrm{CCA}$ specimens from patients admitted at the Second Affiliated Hospital of Harbin Medical University (Harbin, China). Specimens were collected from 2013 to 2018. Histopathological analysis was conducted on the specimens to confirm the diagnosis. Each patient provided informed consent. Patients who had undergone cancer treatment before were not allowed in the present study.

\section{Cell culture and Reagents}

Rabbit monoclonal anti-p53, p21, Beclin-1, p62, CDK4, CDK6, and anti-GAPDH-actin were procured from Abcam (Cambridge, UK). The HIBEC, REB, HCCC-9810, and SSP25 were procured from the Chinese Academy of Sciences (Shanghai, China). Cells were grown (conditions $37^{\circ} \mathrm{C}, 5 \% \mathrm{CO}_{2}$ ) in RPMI-1640/ DMEM augmented with FBS (10\%) and streptomycin/penicillin $(50 \mathrm{mg} / \mathrm{mL} / 100 \mathrm{U} / \mathrm{mL}$, respectively). Less than 6-month old cultures were used to conduct the experiments.

\section{Cell viability assay}

The cholecystokinin-8 (CCK8) kit (Dojindo, Kumamoto, Japan) was applied to examine the viability of the cells. The cells $\left(8 \times 10^{3}\right)$ were seeded onto 96 -well plates 24 hours before transfection was performed. 
Next, we incubated the cells with miR-27a-3p mimic/inhibitor for 48 hours using Lipofectamine 2000 as per the methods described by the manufacturer. These experiments were repeated thrice.

\section{Colony formation assays}

CCA cells were transfected with miR-27a-3p inhibitor or NC group. 48 hours after transfection, 800 cells were counted and seeded into $3.5-\mathrm{cm}$ dishes. After 14 days, colonies were stained with $0.1 \%$ crystal violet in $20 \%$ methanol for 15 minutes. The dishes were photographed and the numbers of visible colonies were counted.

\section{Western blotting analysis}

After protein extraction, the proteins were resolved in 10\% SDS-PAGE. Following blocking, the membranes were incubated together with p-p53, p-21 and GAPDH (1:1000 dilution), Beclin-1, CDK4, CDK6 and p62 (1:500 dilution) antibodies incubated overnight at $4{ }^{\circ} \mathrm{C}$. The bands from the assay were imaged and their intensities were evaluated by Odyssey 3.0 software. $\beta$-actin was used as the reference protein.

\section{Extraction of total RNA and quantitative real-time PCR analysis}

RNAiso Plus reagent (Takara Biotechnology, Dalian, China) was applied in the extraction of total RNA from the cells. After the extraction, reverse transcription was performed using a reverse transcription kit (Takara). Subsequently, RT-PCR was conducted using the primers:

ING5: 5'-AAACGAACCCACGTACTGC-3' (sense) and 5'-TTGCGACACGAATGAAGG-3' (antisense);

GAPDH: 5'-CATTGCCGACAGGATGCA-3' (sense) and

5'-CATCTGCTGGAAGGTGGACAG-3' (antisense). The thermocycler conditions were: initial denaturation for 10 min at $95^{\circ} \mathrm{C}$; then 40 cycles of denaturation for $30 \mathrm{~s}$ at $95^{\circ} \mathrm{C}$, annealing for $15 \mathrm{~s}$ at $55^{\circ} \mathrm{C}$, and extension for $20 \mathrm{~s}$ at $72{ }^{\circ} \mathrm{C}$; a final extension was run for $15 \mathrm{~s}$ at $65^{\circ} \mathrm{C}$. The $2^{-} \Delta \Delta \mathrm{CT}$ method was employed to evaluate expression levels. And GAPDH was used as internal control.

\section{Tumor xenografts in mice}

The athymic BALB/c nude mice used in the present study were procured from the SLRC LaboratoryAnimals (Shanghai, China). And the lentiviral constructs of NC and miR-27a-3p inhibitors were designed and constructed by the Biowit Technology (Shenzhen, China). Seven days after cancer xenografts, each mouse was infected with vectors. Briefly, $10^{6}$ REB cells were intraperitoneally injected into four-week-old female mice. Subsequently, the mice were raised in aseptic conditions for 28 days after which they were sacrificed. For the purpose of immunohistochemical (ki67) staining, the xenograft cancer tissues were extracted from the mice and weighed then fixed using formalin. After fixation, the tissues were embedded in paraffin. The residual cancer tissues were preserved at $-80^{\circ} \mathrm{C}$ for further analysis of the protein and RNA levels. 


\section{Immunofluorescence staining and Immunohistochemistry}

In this procedure, the cells were first put on coverslips then fixed for 20 minutes with $4 \%$ paraformaldehyde. Next, the cells were blocked for 1 hour in BSA $(10 \%)$ then maintained at $4{ }^{\circ} \mathrm{C}$ overnight at together with the ki67 antibody. After that, the cells were maintained at $37^{\circ} \mathrm{C}$ for 1 hour together with the secondary antibody. The cells were assessed by confocal microscopy to determine autophagy. A total of 10 visual fields were selected randomly. Image-Pro Plus 6.0. was used to measure the optical density.

\section{Statistical analysis}

Each experiment was repeated three to six times and presented as the mean \pm standard deviation. Unpaired Student's t-test was used for the parametric data analysis. Values with $\mathrm{P}<0.05$ were considered statistically significant.

\section{Results}

\section{The role of miR-27a-3p in CCA}

To examine the primary role of miR-27a-3p in CCA development, we assessed the miR-27a-3p level in CCA patients by TCGA. The miR-27a-3p level was remarkably overexpressed in CCA tissues relative to adjacent normal tissues (Fig. 1A), and this was correlated with low survival (Fig. 1B). To examine the function of miR-27a-3p in CCA cells, we assessed its level of expression in REB, SSP25 and REB cells which all belong to CCA. It was found the miR-27a-3p was remarkably higher in all CCA cell lines than in the HIBEC (a normal human intrahepatic biliary epithelial cell) (Fig. 1C). For our result of Fig. 1C, we found there is no different among HCCC-9810, SSP25 and REB cells, for further study we use REB cells only.

\section{miR-27a-3p knockdown reduces CCA cells growth}

The effect of miR-27a-3p on CCA tumorigenicity was assessed by treating REB cells with NC/miR-27a-3p inhibitors. The colony formation and CCK-8 revealed that knockdown of miR-27a-3p inhibited REB cell growth (Fig. 2A-C). Furthermore, immunofluorescence staining showed a significantly lower Ki-67 signal in the miR-27a-3p knockdown group relative to the NC group (Fig. 2D). Consistently, the levels of CDK4 and CDK6 also decreased whereas p-p53 and p21 levels were increased due to miR-27a-3p knockdown than those in the NC group (Fig. 2E, F).

To assess the miR-27a-3p effect on CCA in vivo, we constructed a nude mouse xenograft cancer model by subcutaneous inoculation of REB transfected with NC/ miR-27a-3p inhibitors. As expected, the size and the weight of the xenograft tumor in the miR-27a-3p knockdown group were considerably smaller relative to that of the NC group (Fig. 2G-I). In addition, a much lower Ki-67 level in xenograft specimens was observed in the miR-27a-3p inhibitor transfected REB group relative to the NC group (Fig. 2G). 
The findings presented here implied that depletion of miR-27a-3p reduces CCA cell proliferation capability.

\section{miR-27a-3p knockdown enhances autophagy induction in CCA cells}

Autophagy, an intracellular catabolic process, provide energy and macromolecular precursors for cell growth in many diseases[14]. Herein, transmission electron microscope (TEM) was used to test the autophagy corpuscle in REB cells after treat with miR-27 inhibitor. Our data showed silence of miR-27a-3p induce autophagy in REB cells (Fig. 3A). Then, we test the protein level of beclin-1 and p62 after transfect with miR-27a-3p inhibitor. Our results showed miR-27a-3p knockdown remarkably induced Beclin-1 level and accumulated p62 level in REB cells compared to NC groups indicating that miR-27a-3p mediated autophagy in CCA cells (Fig. 3B). Further, it was observed that miR-27a-3p depletion promoted the conversion of LC3-II/LC3-I (Fig. 3C). To strength our data, the autophagic flux was examined by the tandem-tagged LC3 construct mRFP-GFP-LC3. REB cells with miR-27a-3p silencing tended to be red. However, inhibitor-NC transfected cells predominantly in yellow. These observations demonstrated that miR-27a-3p knockdown promotes autophagy induction in CCA cells.

\section{ING5 directly target miR-27a-3p}

miRNA is involved in the pathophysiological process in many diseases by negative regulation of target genes[15].To found the potential target gene of miR-27a-3p in CCA, we used two independent algorithms, TargetScan (www.targetscan.org/), and mirbase (www.mirbase.org/), and found that ING5 could be a potential gene of miR-27a-3p target (Fig. 4A). We next determined the expression protein and mRNA levels of ING5 after transfection with miR-27a-3p mimics. Results indicated that mRNA level of ING5 was lower than the NC group after miR-27a-3p-mimic transfection, and western blot assay indicated a similar pattern of ING5 protein level. MiR-27a-3p inhibitor remarkably up-regulated ING5 in mRNA as well as protein levels (Fig. 4B-C) $[\mathrm{P}<0.05]$. Based on the above results, we think ING5 maybe a possible miR-27a$3 p$ target.

Luciferase reporter assay was conducted to determine if miR-27a-3p directly targets ING5. The miR-27a$3 p$ mimics suppressed luciferase activity in the ING5-WT group rather than the ING5-Mut group (Fig. 4D). Hence, we believed that miR-27a-3p direct targeted ING5 in CCA.

\section{Over-expression of ING5 enhances autophagy induction in CCA cells}

A prior study showed ING5 induces autophagy in gastric cancer cells [16]. However, there is no evidence showed ING5 can regulate autophagy in CCA. We first test the expression of ING5 in CCA cells after transfect with ING5 overexpression. We found the expression of ING5 is up-regulate in CCA cells (Figure S1). The TEM was employed to test the autophagy corpuscle in CCA cells after treat with ING5 overexpression (Fig. 5A). And then we test the expression of autophagy-relative proteins. Our data showed 
that overexpression of ING5 can remarkably induced Beclin-1 level and accumulated p62 level in REB cells compared to NC groups indicating that ING5 mediated autophagy in CCA cells (Fig. 5B). Further, it was observed that ING5 overexpression promoted the conversion of LC3-II/LC3-I (Fig. 5C). Our data demonstrated that up-regulate ING5 promotes autophagy induction in REB cells.

\section{The effects of miR-27a-3p were eliminated by ING5}

To further demonstrate that ING5 mediated miR-27a-3p to enhance tumor growth, we evaluated the effect of miR-27a-3p on the ability of REB cells to proliferate by ING5-siRNA. The results of the CCK8 assay indicated that the proliferation of the cells inhibited by miR-27a-3p inhibitor relative to the inhibitor NC group. But, the co-transfection of ING5 siRNA at least partially reversed growth inhibition caused by miR27a-3p knockdown (Figure. 6A). And also, a similar phenotype was observed by colony formation assay and KI67 staining (Figure. 6B-C). Further, we found that the co-transfection of ING5 siRNA restored the cell apoptosis induced by miR-27a-3p inhibitor (Fig. 6D). The findings of the current study suggested that miR-27a-3p could partially enhance the proliferation of REB cells by direct targeting ING5.

\section{Discussion}

CCA is the second most prevalent primary hepatobiliary malignancy worldwide[17]. Despite several medical studies on the condition, CCA treatment/management still poses a serious challenge to public health[18]. The latest findings have suggested that miRNAs could play an essential role in CCA development[19]. These studies laid the foundation for exploring the molecular mechanisms underlying the CCA development. Herein, it was revealed that miR-27a-3p silencing could inhibit the growth and induce autophagy of CCA cells. miR-27a-3p exerted its partial oncogenic effect by interacting with the autophagy signaling pathway, thus suppressing the ING5 gene expression.

miRNA is able to target direct mRNA and modulate down-stream gene expression post-transcriptionally, can regulated in many diseases, such as miR-27a-3p $[20,21]$. The miRNA promotes the progression of gastric cancer by BTG2 regulation [22]. miR-27a-3p has been identified as marker of the recurrence of clear cell renal cell carcinoma[23]. According to the results herein, high miR-27a-3p mRNA levels was observed in CCA tissues and in cell lines (Figure 1). MiR-27a-3p silencing inhibited CCA cell proliferation and induce apoptosis (Figure 2). Our results were consistent with other studies which also verified that miR-27a-3p possesses excellent oncogenaic effects in CCA.

Autophagy has a crucial function in the formation and development of several diseases, such as CCA $[9$, $24,25]$. Autophagy occurs to maintain cell balance [26] . The process occurs to ensure the survival of cells amid environmental stress. But in the process, autophagy seems to enhance cell morbidity and death [27] .Evidence shows that abnormal autophagy signaling pathway activation contributes to cancer development. Ma et al showed that miR-124 could trigger autophagy-associated apoptosis in CCA cells by regulating the EZH2-STAT3 signaling axis.[9] But studies are yet to reveal whether miR-27a-3p exercises its oncogenic function by autophagy signaling pathway regulation in CCA. Herein, we show that miR-27a-3p inhibition promotes the ratio of LC3 II and LC3 I, up-regulate the autophagy relative protein 
beclin-1 and p62 expression. Immunofluorescence examination revealed that cells in which miR-27a-3p was silenced exhibited lower-expression relative to the inhibitor-NC group, suggesting that miR-27a-3p knockdown promoted autophagy induction in CCA cells.

ING5 has been identified as a tumor inhibitor in numerous human cancer diseases [28] [29]. Besides, ING5 is downregulated in several cancers, thus suppressing its function of inhibiting the autophagy signaling pathway[30].Herein, miR-27a-3p inhibition can induce autophagy. Over-expression ING5 also promote autophagy in REB cells. These data suggesting that miR-27a-3p probably activates the autophagy signaling pathway through suppressing ING5. Thus, the ING5 function in CCA might not be restricted to autophagy signaling pathway inhibition. Also, MiR-27a-3p might suppress the transcriptional role of autophagy through ING5.

In summary, we revealed that miR-27a-3p suppression triggered apoptosis and suppressed cell proliferation by regulating cell autophagy in human CCA cells. Thus, the partial oncogenic role of the gene in CCA cells could be through its regulation of the autophagy signaling pathway via directly targeting ING5. Our findings may provide a novel insight into miR-27a-3p in CCA progression, and the gene could be a possible CCA treatment target.

\section{Declarations}

\section{Ethics approval and consent to participate}

Not applicable

\section{Consent for publication}

Not applicable

\section{Availability of data and materials}

The datasets used and/or analysed during the current study are available from the corresponding author on reasonable request.

\section{Competing interests}

The authors declare that they have no competing interests.

\section{Funding}

Not applicable

\section{Authors' contributions}

MW and YF C: Conceptualization, Methodology, Software. 
FM Z, PC K and XM J: Original draft preparation.

All authors read and approved the final manuscript.

\section{Acknowledgements}

Not applicable

\section{References}

1. Banales JM, Cardinale V, Carpino G, Marzioni M, Andersen JB, Invernizzi P, Lind GE, Folseraas T, Forbes SJ, Fouassier L. Expert consensus document: Cholangiocarcinoma: current knowledge and future perspectives consensus statement from the European Network for the Study of Cholangiocarcinoma (ENS-CCA). Nature reviews Gastroenterology hepatology. 2016;13:261.

2. Razumilava N, Gores GJ: Cholangiocarcinoma. The Lancet. 2014;383:2168-79.

3. Rizvi S, Khan SA, Hallemeier CL, Kelley RK, Gores GJ. Cholangiocarcinoma-evolving concepts and therapeutic strategies. Nature Reviews Clinical Oncology. 2018;15:95.

4. Massarweh NN, El-Serag HB. Epidemiology of hepatocellular carcinoma and intrahepatic cholangiocarcinoma. Cancer Control. 2017;24:1073274817729245.

5. Liang H, Su X, Wu Q, Shan H, Lv L, Yu T, Zhao X, Sun J, Yang R, Zhang L, et al: LncRNA 2810403D21Rik/Mirf promotes ischemic myocardial injury by regulating autophagy through targeting Mir26a. Autophagy 2019:1-15.

6. D'Adamo S, Cetrullo S, Guidotti S, Silvestri Y, Minguzzi M, Santi S, Cattini L, Filardo G, Flamigni F, Borzi RM. Spermidine rescues the deregulated autophagic response to oxidative stress of osteoarthritic chondrocytes. Free Radic Biol Med 2020.

7. Perez-Montoyo H. Therapeutic Potential of Autophagy Modulation in Cholangiocarcinoma. Cells 2020, 9.

8. Lv P, Luo YF, Zhou WY, Liu B, Zhou Z, Shi YZ, Huang R, Peng C, He ZL, Wang J, et al: miR-373 inhibits autophagy and further promotes apoptosis of cholangiocarcinoma cells by targeting ULK1. Kaohsiung J Med Sci 2020.

9. Ma J, Weng L, Wang Z, Jia Y, Liu B, Wu S, Cao Y, Sun X, Yin X, Shang M, Mao A. MiR-124 induces autophagy-related cell death in cholangiocarcinoma cells through direct targeting of the EZH2-STAT3 signaling axis. Exp Cell Res. 2018;366:103-13.

10. Zhang T, Meng J, Liu X, Zhang X, Peng X, Cheng Z, Zhang F. ING5 differentially regulates protein lysine acetylation and promotes p300 autoacetylation. Oncotarget. 2018;9:1617.

11. Barlak N, Capik O, Sanli F, Kilic A, Aytatli A, Yazici A, Ortucu S, Ittmann M, Karatas OF. ING5 inhibits cancer aggressiveness by inhibiting Akt and activating p53 in prostate cancer. Cell Biol Int. 2020;44:242-52. 
12. Cui S, Liao X, Ye C, Yin X, Liu M, Hong Y, Yu M, Liu Y, Liang H, Zhang C-Y. ING5 suppresses breast cancer progression and is regulated by miR-24. Mol Cancer. 2017;16:89.

13. Zhao QY, Ju F, Wang ZH, Ma XZ, Zhao H. ING5 inhibits epithelial-mesenchymal transition in breast cancer by suppressing PI3K/Akt pathway. Int J Clin Exp Med. 2015;8:15498-505.

14. Wang D, Guo H, Yang H, Wang D, Gao P, Wei W. Pterostilbene, An Active Constituent of Blueberries, Suppresses Proliferation Potential of Human Cholangiocarcinoma via Enhancing the Autophagic Flux. Front Pharmacol. 2019;10:1238.

15. Zhang JW, Wang X, Li GC, Wang D, Han S, Zhang YD, Luo CH, Wang HW, Jiang WJ, Li CX, Li XC. MiR30a-5p promotes cholangiocarcinoma cell proliferation through targeting SOCS3. J Cancer. 2020;11:3604-14.

16. Gou WF, Shen DF, Yang XF, Zhao S, Liu YP, Sun HZ, Su RJ, Luo JS, Zheng HC. ING5 suppresses proliferation, apoptosis, migration and invasion, and induces autophagy and differentiation of gastric cancer cells: a good marker for carcinogenesis and subsequent progression. Oncotarget. 2015;6:19552-79.

17. Krasinskas AM. Cholangiocarcinoma. Surg Pathol Clin. 2018;11:403-29.

18. Wang Y, Wan M, Zhou Q, Wang H, Wang Z, Zhong X, Zhang L, Tai S, Cui Y. The Prognostic Role of SOCS3 and A20 in Human Cholangiocarcinoma. PLoS One. 2015;10:e0141165.

19. Labib PL, Goodchild G, Pereira SP. Molecular Pathogenesis of Cholangiocarcinoma. BMC Cancer. 2019;19:185.

20. Chen Q, Liu T, Bao Y, Zhao T, Wang J, Wang H, Wang A, Gan X, Wu Z, Wang L. CircRNA cRAPGEF5 inhibits the growth and metastasis of renal cell carcinoma via the miR-27a-3p/TXNIP pathway. Cancer Lett. 2020;469:68-77.

21. Ding F, Jiang K, Sheng Y, Li C, Zhu H. LncRNA MIR7-3HG executes a positive role in retinoblastoma progression via modulating miR-27a-3p/PEG10 axis. Experimental Eye Research 2020:107960.

22. Zhou L, Liang X, Zhang L, Yang L, Nagao N, Wu H, Liu C, Lin S, Cai G, Liu J. MiR-27a-3p functions as an oncogene in gastric cancer by targeting BTG2. Oncotarget. 2016;7:51943-54.

23. Li Y, Li J, Sun X, Chen J, Sun X, Zheng J, Chen R. MicroRNA-27a functions as a tumor suppressor in renal cell carcinoma by targeting epidermal growth factor receptor. Oncol Lett. 2016;11:4217-23.

24. Wang Y, Jiang W, Li C, Xiong X, Guo H, Tian Q, Li X. Autophagy Suppression Accelerates Apoptosis Induced by Norcantharidin in Cholangiocarcinoma. Pathol Oncol Res 2019.

25. Thongchot S, Vidoni C, Ferraresi A, Loilome W, Yongvanit P, Namwat N, Isidoro C. Dihydroartemisinin induces apoptosis and autophagy-dependent cell death in cholangiocarcinoma through a DAPK1BECLIN1 pathway. Mol Carcinog. 2018;57:1735-50.

26. Xu Y, Wan W. The bifunctional role of TP53INP2 in transcription and autophagy. Autophagy 2020:13.

27. Tang Z, Zhang Z, Zhang H, Wang Y, Zhang Y, Zhao J, Yang H, Wang Z. Autophagy Attenuation Hampers Progesterone Synthesis during the Development of Pregnant Corpus Luteum. Cells $2019,9$. 
28. Gao W, Han J. Overexpression of ING5 inhibits HGF-induced proliferation, invasion and EMT in thyroid cancer cells via regulation of the c-Met/PI3K/Akt signaling pathway. Biomed Pharmacother. 2018;98:265-70.

29. Archambeau J, Blondel A, Pedeux R. Focus-ING on DNA Integrity: Implication of ING Proteins in Cell Cycle Regulation and DNA Repair Modulation. Cancers (Basel) 2019, 12.

30. Zheng HC, Zhao S, Song Y, Ding XQ. The roles of ING5 expression in ovarian carcinogenesis and subsequent progression: a target of gene therapy. Oncotarget. 2017;8:103449-64.

\section{Figures}

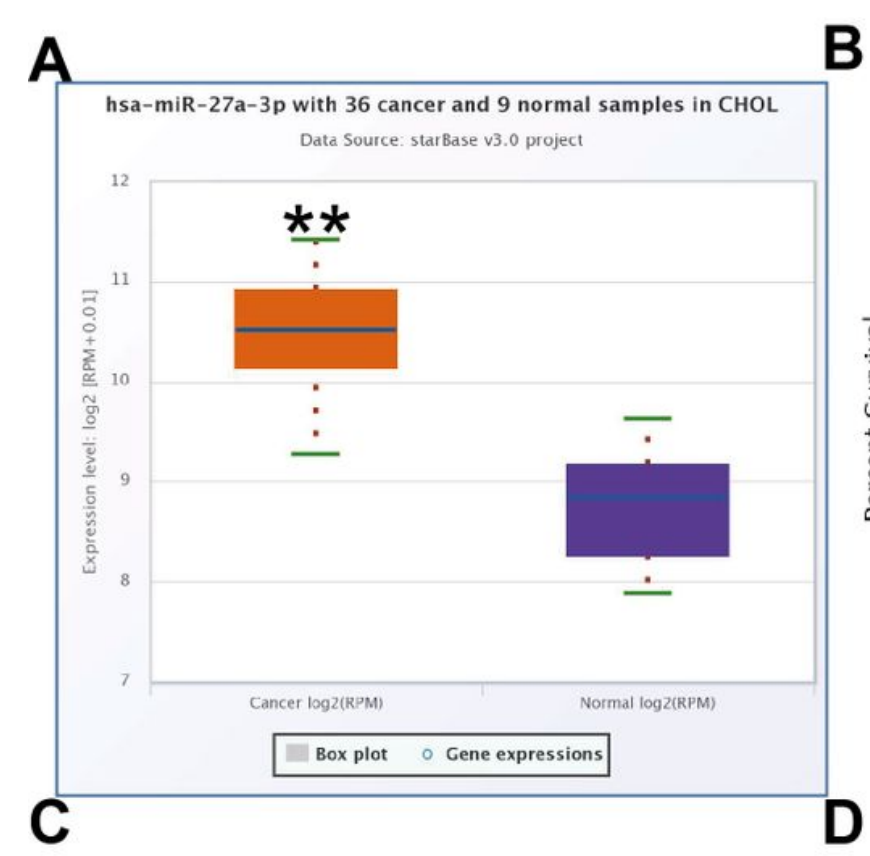

\section{$\mathbf{B}$}
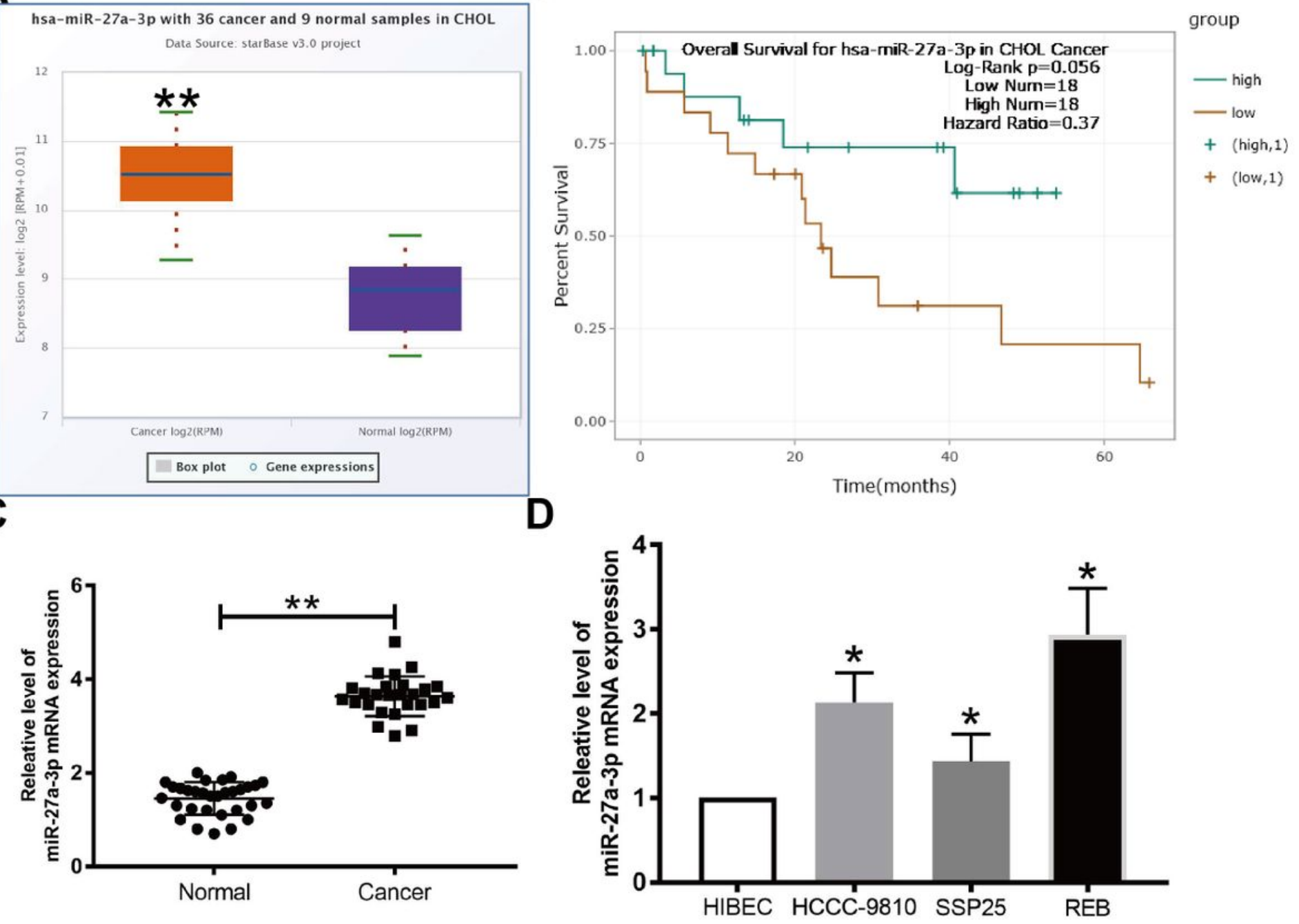

\section{Figure 1}

The expression of miR-27a-3p in CCA. GEO dataset showed the expression of miR-27a-3p in human CCA tissues and their matched noncancerous tissues(A). (B)The survival of miR-27a $3 p$ in CCA patients. (C-D) Relative miR-27a-3p mRNA expression levels in CCA tissue and HIBE and CCA cell lines. ${ }^{*} P<0.05$ versus normal group or HIBE group. 
A

B
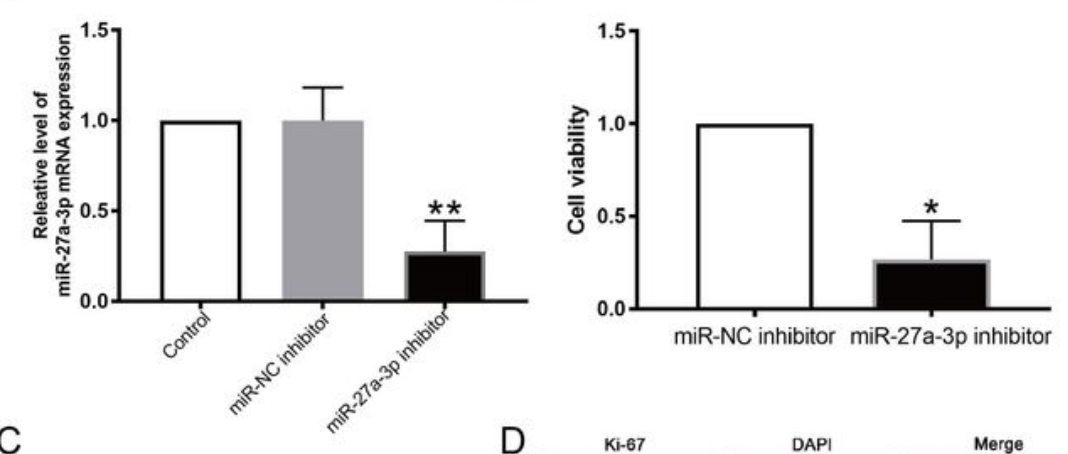

C

miR-NC inhibitor

miR-27a-3p inhibitor

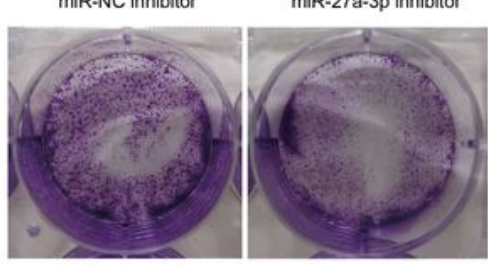

D Ki-67
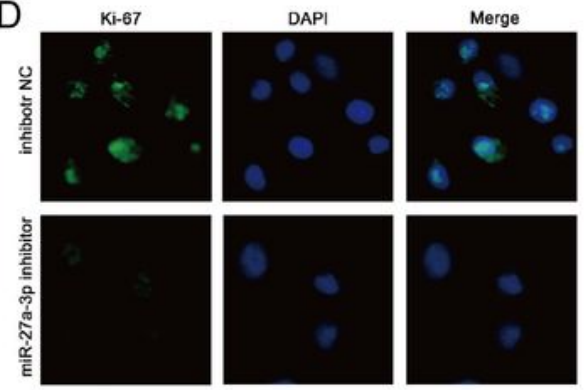

E
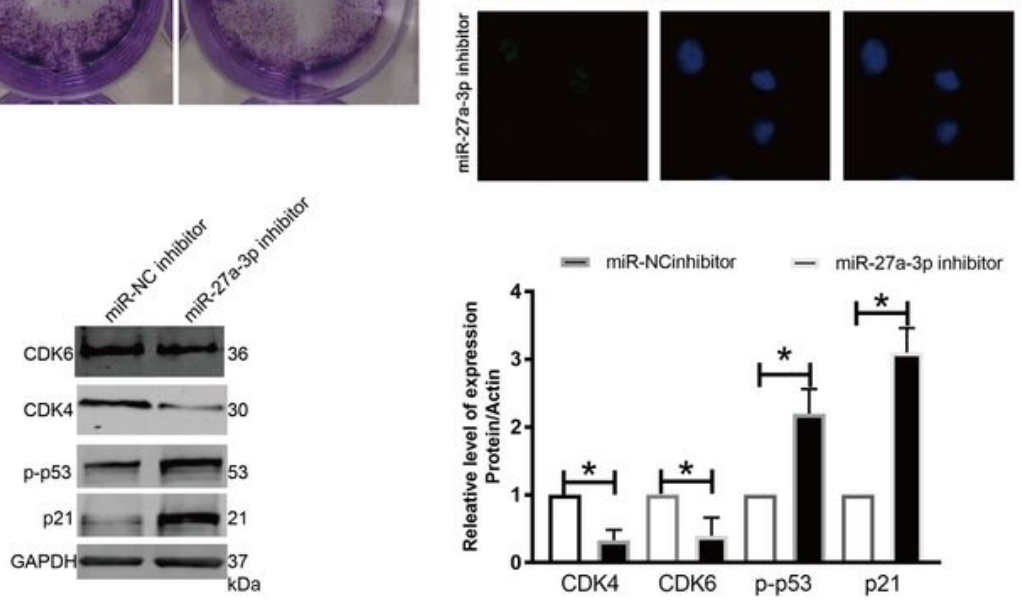

F

G
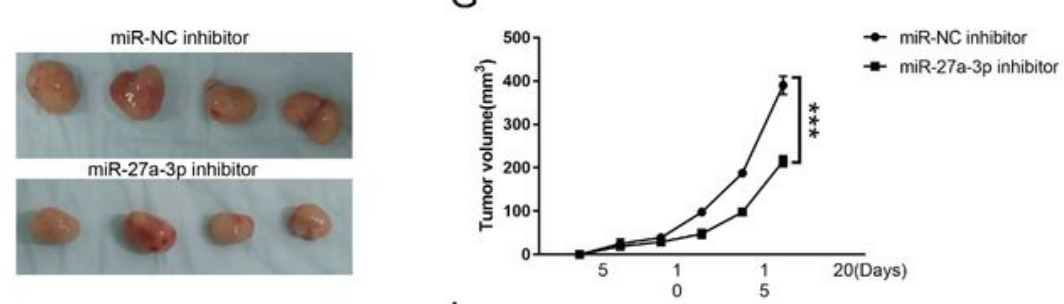

$\mathrm{H}$
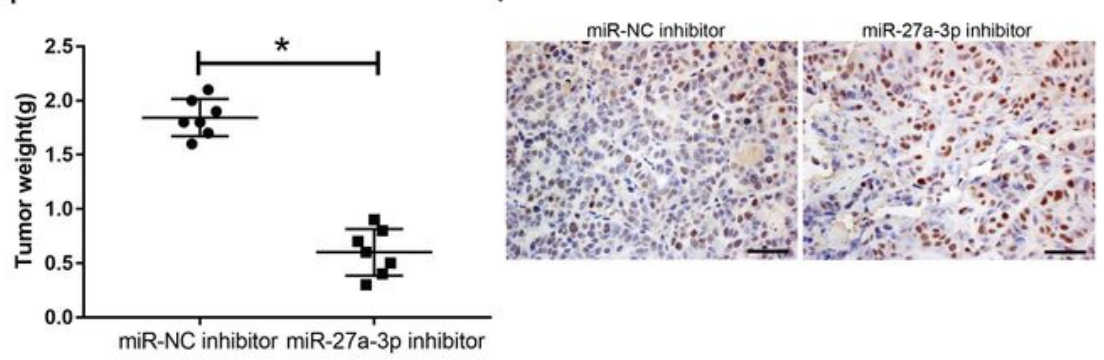

Figure 2

miR-27a-3p knockdown reduces CCA cells growth $(A)$ The expression of miR-27a-3p after transfect with miR-27a-3p inhibitor. (B) Cell variability was tested by CCK-8 after treatment with miR-27a-3p inhibitor or miR-inhibitor NC in REB cells. (C-D) Colony formation and Ki67 assay showed cell proliferation after the treatment of miR-27a-3p inhibitor or miR-inhibitor NC. (E) The protein level of CDK4, CDK6, p-p53 and p21 were measured by western blotting following transfect with miR-27a-3p inhibitor. (F-I) The tumor growth 
measured in the nude mouse xenograft tumor model after transfection with miR-27a-3p. Error bars show the mean \pm SD of three experimental repeats. ${ }^{*} \mathrm{P}<0.05 \mathrm{vs}$. miR-inhibitor $\mathrm{NC}$ group.

A

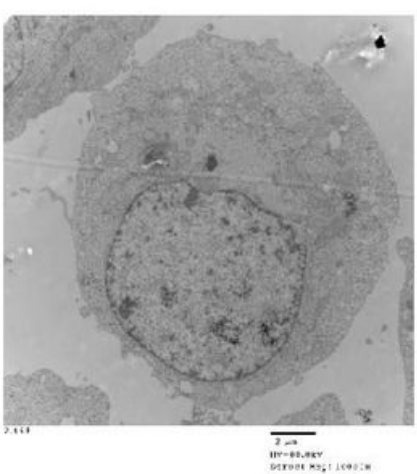

miR-NC inhibitor

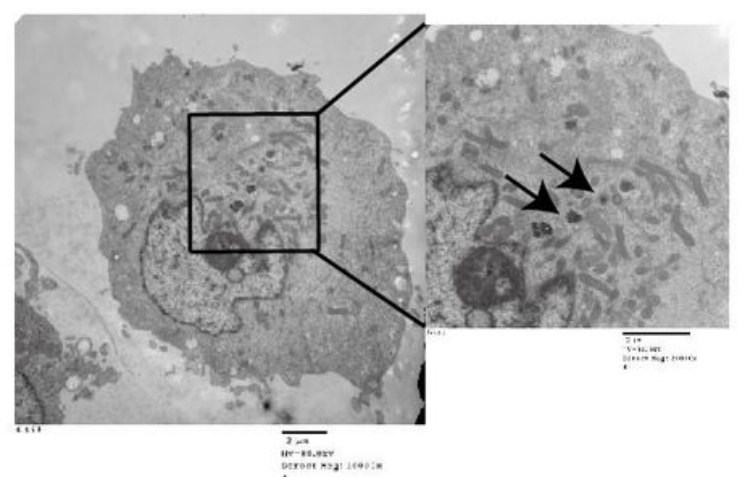

miR-27a-3p inhibitor

B
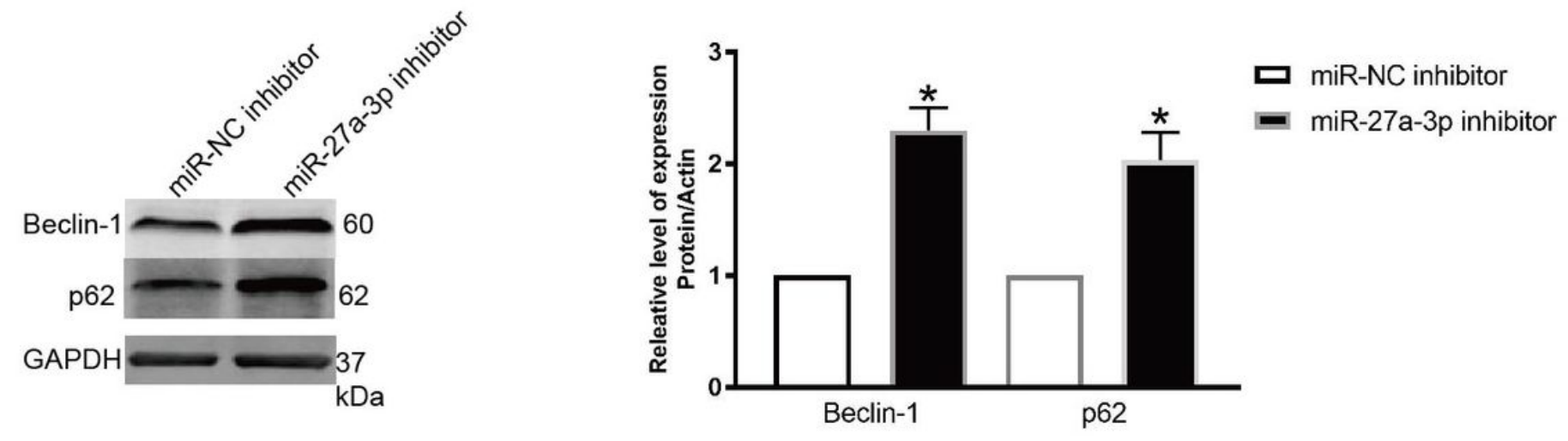

C

miR-NC inhibitor

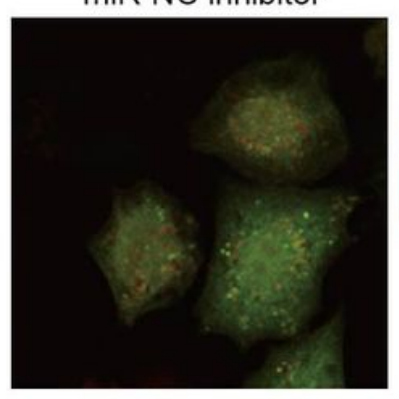

miR-27a-3p inhibitor

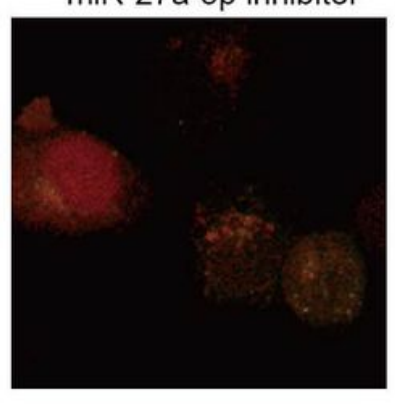

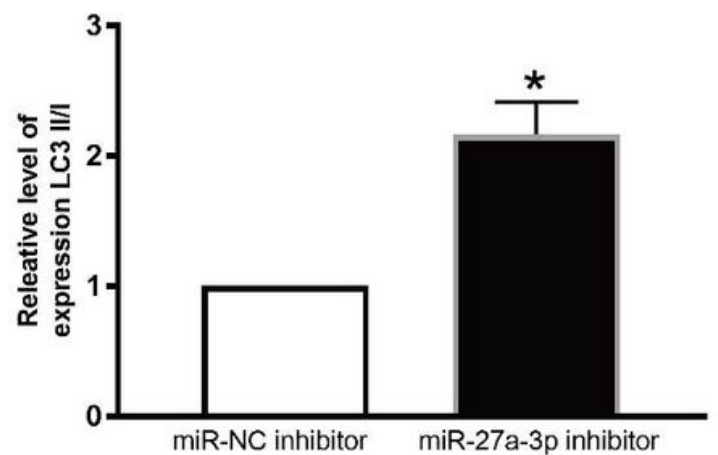

Figure 3

miR-27a-3p knockdown promotes autophagy induction in CCA cells. Notes: (A) Autophagy corpuscle was tested by TEM after transfects with miR-27a-3p inhibitor. (B) Protein level of Beclin-1 and p62 were test by western blotting after treatment with miR-27a-3p inhibitor/ miR-inhibitor NC in REB cells. (C) LC3 II/I assay showed the cell autophagy. Error bars show the mean $\pm S D$ of 3 experimental repeats. ${ }^{*} P<0.05$ vs. miR-inhibitor NC group. 
A

\section{ING5-WT 5'..AAUUGGUGAUUUUUUACUGUGAA... \\ | | | | | | | | \\ miR-27a-3p 3' CGCCUUGAAUCGGUGACACUU}

ING5-Mut 5'..AAUUGGUGAUUUUUUGCACGCGG...

B
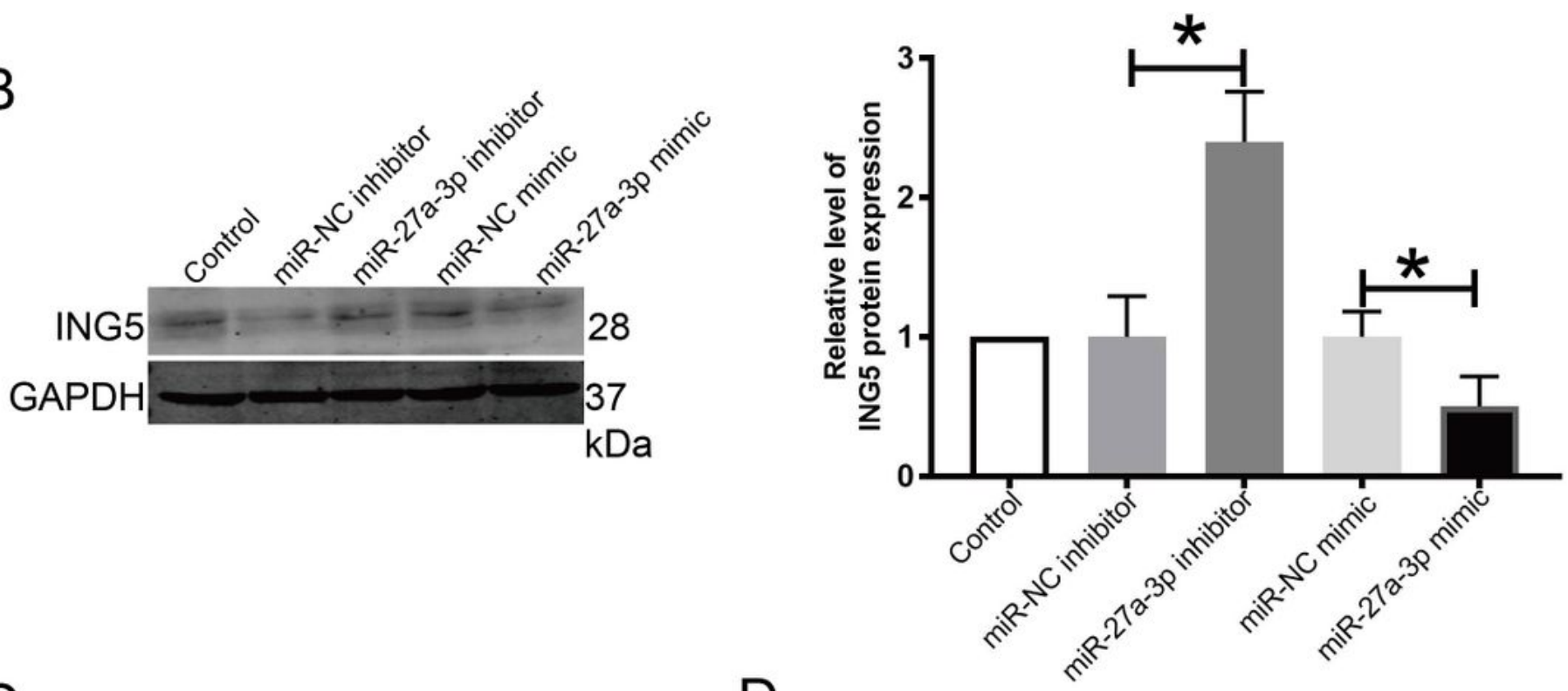

C
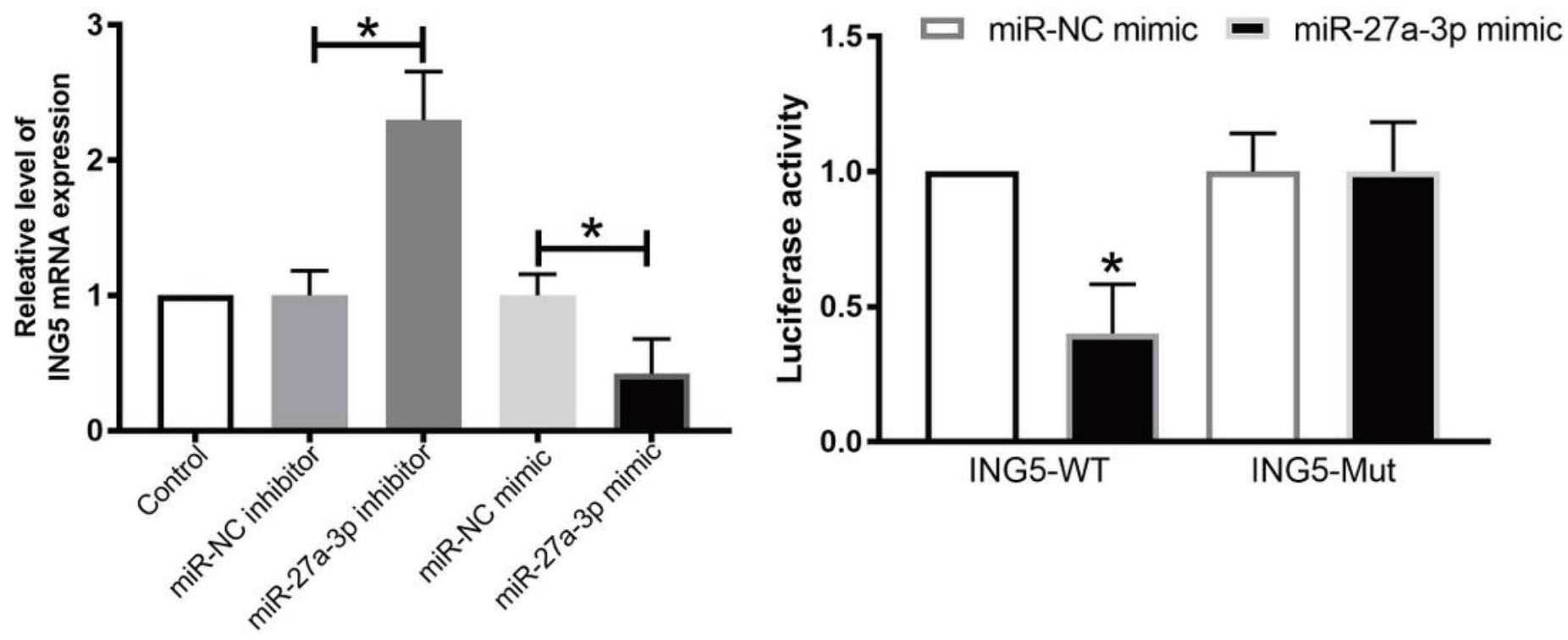

Figure 4

ING5 is a direct miR-27a-3p target (A) Binding site in miR-27a-3p and the 3'-UTR of the ING5 gene. (B) Relative ING5 mRNA expression after treatment with miR-27a-3p mimic, miR-mimic NC or miR-27a-3p inhibitor, miR-inhibitor NC by qRT-PCR. (C) The expression of ING5 after treatment of miR-27a-3p mimic, miR-mimic NC or miR-27a-3p inhibitor, miR-inhibitor NC by western blotting. (D) The luciferase activity of 
ING5 3'-UTR mutant construct regulated by miR-27-3p. (Error bars represent means \pm SEM, $n=3$ for every group. ${ }^{*} \mathrm{P}<0.05$ vs. miR-mimic NC group or miR-inhibitor NC group.

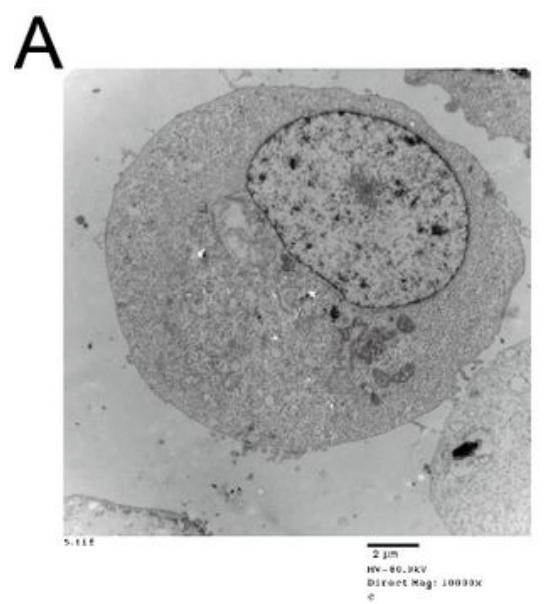

Empty vetor

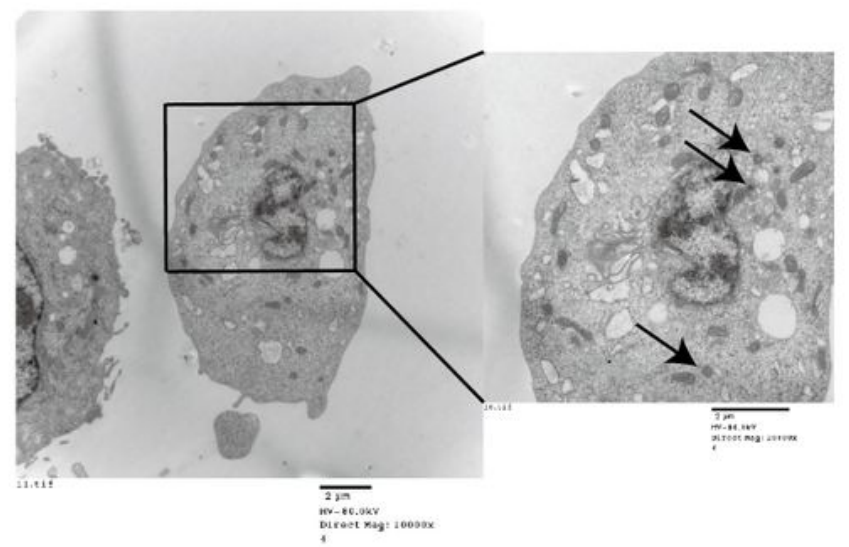

ING5

B
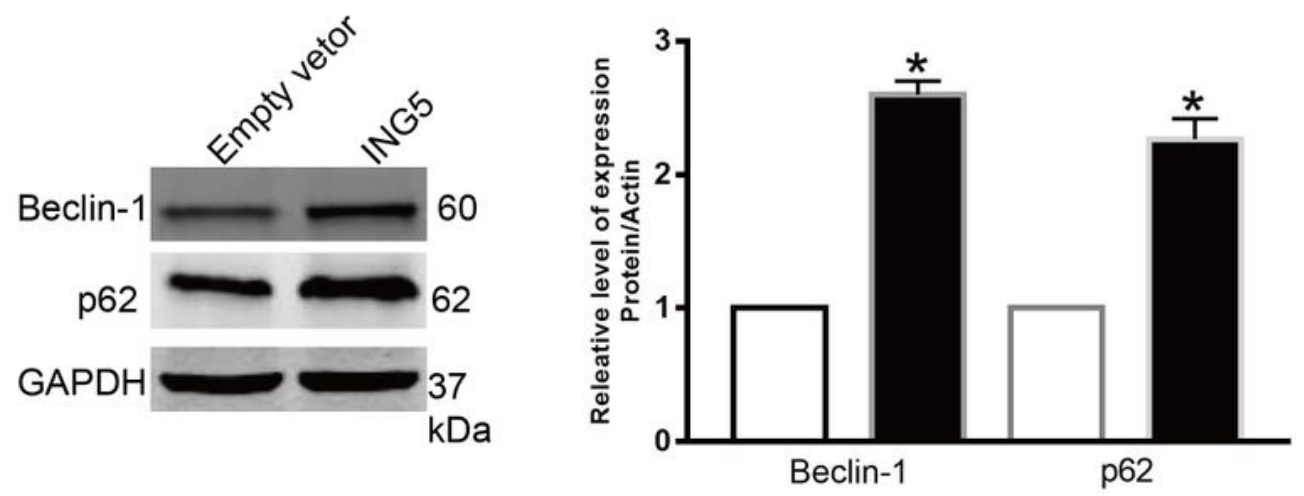

$\square$ Empty vetor

- ING5

$\mathrm{kDa}$

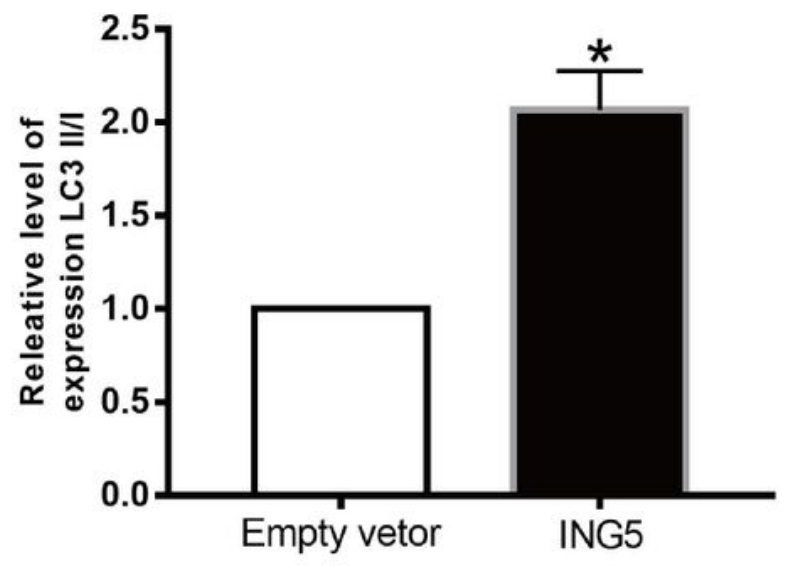

Figure 5

Over-expression ING5 promotes autophagy induction in REB cells. Notes: (A) Autophagy corpuscle was tested by TEM after transfects with ING5 overexpression. (B) Protein level of Beclin-1 and p62 were test by western blotting after treatment with ING5 overexpression / empty vector in REB cells. (C) LC3 II/I 
assay showed the cell autophagy. Error bars show the mean \pm SD of 3-6 experimental repeats. *P<0.05 vs. empty vector group.

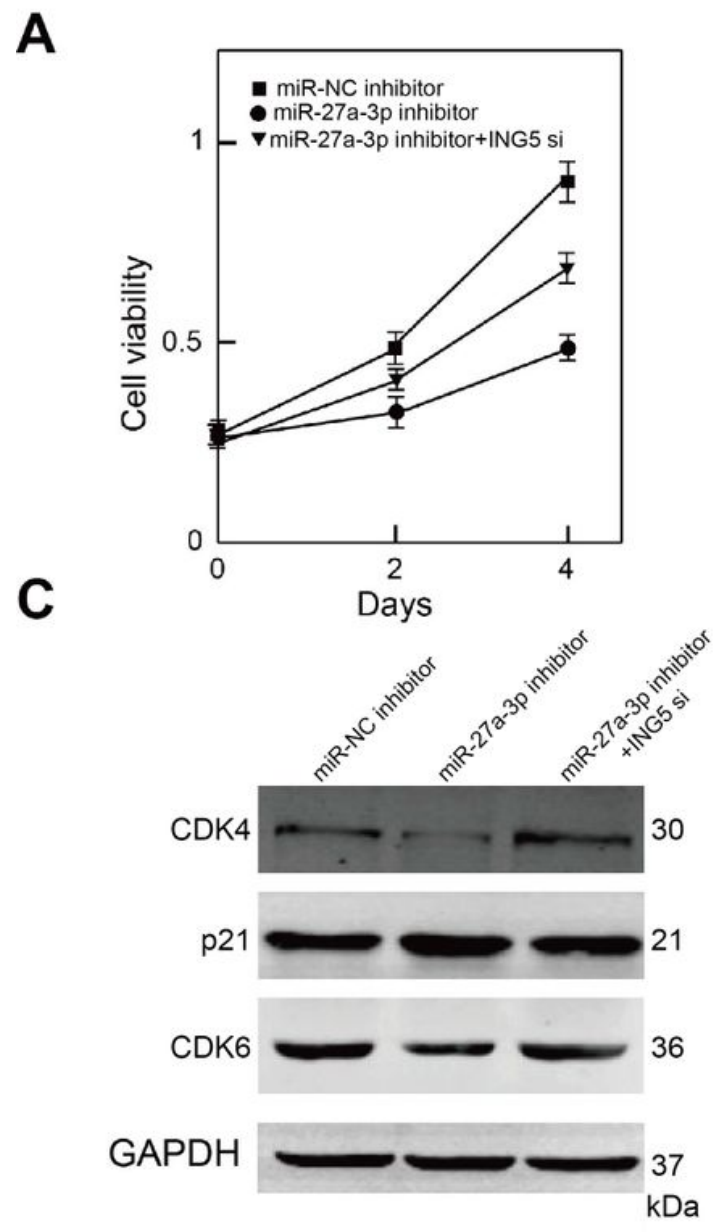

B

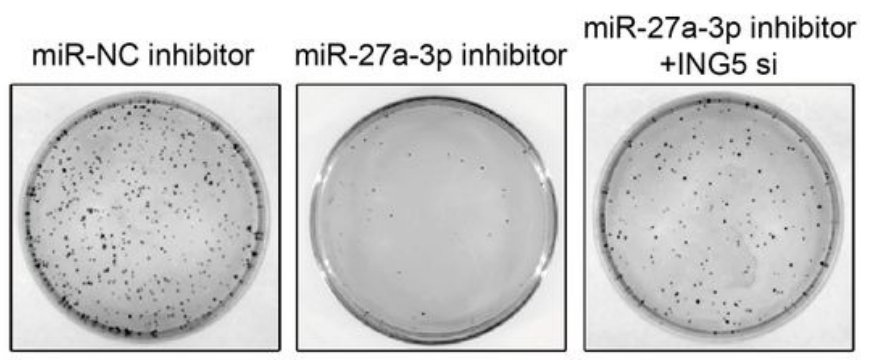

\section{Figure 6}

The effects of miR-27a-3p were eliminated by ING5. Notes: REB cells were treated with miR-27a-3pinhibitor, miR-inhibitor NC or miR-27a-3p-inhibitor+si-ING5 for 48 hours. (A) Cell viability was test by CCK8. (B) colony formation assay, (C) protein levels of CDK4, CDK6 and p21. All experiments were repeated three times. Each bar represents the mean \pm standard deviation of three independent experiments. ${ }^{*} \mathrm{P}<0.05$ versus miR-mimic NC group

\section{Supplementary Files}

This is a list of supplementary files associated with this preprint. Click to download.

- FigureS1.jpg 